\title{
Topiramate Adjunctive Therapy for Olanzapine Induced Weight Gain in Patients with Schizophrenia
}

\section{*Sanjib Pandit ${ }^{1}$, Asha Sharma ${ }^{2}$}

${ }^{1}$ Lecturer, Department of Psychiatry, Karnali Academy of Health Sciences Jumla Nepal

${ }^{2}$ Medical Officer, Karnali Academy of Health Sciences Jumla Nepal

\begin{abstract}
Background: In recent days, the prevailing use of second-generation antipsychotics (SGA) or atypical antipsychotics over conventional antipsychotics have shifted the concern of physician from extrapyramidal side effects to the weight gain in the patients receiving treatment for schizophrenia. Among others atypical anti-psychotics, the phenomenology of Olanzapine related weight gain is highly recognized in the clinical practice. However, the exact mechanism by which Olanzapine exerts weight gain effect is largely not understood and is very likely to be multi-factorial. Among other several risk factors, factors such as 5HT2c polymorphisms and H1 receptor affinity have also been purposed. Similarly, various neuro-endocrinal factors related in maintaining energy homeostasis have also been observed to be affected by Olanzapine treatment. However, no consistent findings are available to clearly explain the underlying psychopathology of disease spectrum in schizophrenia. However, though, recent finding from a clinical trial carried out in healthy male volunteers have suggested that the low baseline TSH profile predicts Olanzapine related weight gain and also interestingly relief by Topiramate adjuvant therapy. However, no such investigations were found in a schizophrenic patient.
\end{abstract}

Keywords: Topiramate, Olanzapine, Weight Gain, Schizophrenia

\section{*Corresponding Author:}

\section{Dr. Sanjib Pandit}

Email: panditsanjib@outlook.com

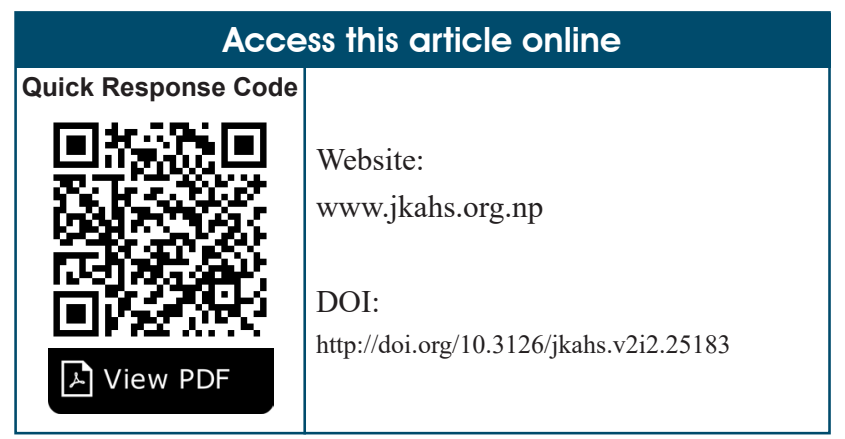

\section{Article Info.}

How to cite this article?

Pandit S, Sharma A. Topiramate Adjunctive Therapy for Olanzapine Induced Weight Gain in Patients with Schizophrenia. Journal of Karnali Academy of Health Sciences. 2019;2(2):147-150

Received: 1 May, Accepted: 21 July, Published: 30 August 2019

Conflict of Interest: None, Source of Support: None

\section{INTRODUCTION}

For the present day, the atypical antipsychotics or the second generation antipsychotics (SGAs) treatment for psychotic like symptoms remains the first line drug treatment for the schizophrenia patients. Among other, Olanzapine is long proved to be an effective medication well known for its clinical efficacy and tolerability. ${ }^{1}$ Olanzapine is long recognized for its effectiveness in reduction of general psychopathology and disease symptoms, a long duration of successful treatment, and low rates acute exacerbation and further reducing the number of re-hospitalization in patients receiving Olanzapine treatment for schizophrenia. ${ }^{1,}$ ${ }^{2}$ However, it is also well evident that its use is limited by the adverse metabolic side effects such as hyperglycemia, hyper-lipidemia, hyper-insulinemia along with weight gain. ${ }^{3}$ 
Topiramate, which is primarily used in epilepsy and migraine prophylaxis, is reportedly found to have weight loss effect in patients receiving its treatment. Several researches afterwards have explored its effectiveness in various other conditions including bipolar affective disorder and binge eating disorder with obesity. The effectiveness of Topiramate treatment for atypical antipsychotic and Olanzapine related treatment in schizophrenia patient has been demonstrated in several randomized controlled trials. And above to all, the use of adjunctive Topiramate treatment for Olanzapine induced weight gain not only resulted in weight loss in these patients but also resulted in improvement of insulin sensitivity, cholesterol level, triglyceride level including improvement in overall symptomatology. Additionally, since this is not the case that the entire schizophrenia patient taking Olanzapine gain weight, some are even found to lose weight while taking Olanzapine, ${ }^{4}$ the prospective marker to define weight gain in this population have been of clinical importance. Defining such markers and evaluating the effectiveness of adjunctive Topiramate treatment to counteract these adverse effects will not only benefit on a metabolic level, it will also help to avoid or decrease the occurrence of unwanted side effects, either by excluding those are at risk or treating with adjuvant selected therapy.

\section{DISCUSSION}

Olanzapine induced body weight gain can be remarkably large and can become distressing to those individuals who experience it. Weight gain in itself further increases the risk of diabetes mellitus while individuals suffering from schizophrenia are already known to be pre-disposed to develop diabetes regardless the administration of any of anti-psychotic treatment. And it is also well known information that, metabolic syndrome and cardiovascular diseases are important causes of both the morbidity and the mortality among patients with severe type of mental illnesses including schizophrenia. However, the use of Olanzapine is strengthen with the fact that, it is among the most effective treatments in psychosis according to a meta-analysis of randomized controlled trials, 1 and may be even more so in a real-life setting as it is also known to reduce exacerbation and re-hospitalization in schizophrenia patient receiving its treatment. ${ }^{2}$

In a recent meta-analysis of 32 studies including 1482 subjects, 15 different weight reducing medications were tested; among which Metformin, Sibutramine, Topiramate, and Reboxetine were found to be superior to the placebo, and Metformin outperformed over other agents, including Topiramate. ${ }^{5}$ However, the evidence is too limited to support Metformin as an adjunctive medication in regular clinical practice. And it is also be noticed that the data regarding Metformin, although, rare but it's life threatening side effects such as lactic acidosis specially in the elderly people and people with renal function impairment, ${ }^{6}$ along with its newly found association regarding accumulation of beta-amyloid factor responsible for the pathogenesis of Alzheimer's disease, ${ }^{7}$ puts on a dilemma in the selection of therapy during clinical practice. It is also evident that the weight loss effect of Metformin is significant only in those patient who already have experienced it and the effect was minimal when started together with anti-psychotics treatment. Whereas, Topiramate in other hand, seems to benefit in either condition; whether to patients who are newly initiated as the preventive or as the attenuation for those who already experienced weight gain. Similarly, in a randomized double blind placebo controlled study conducted in India, Topiramate was given to the first episode drug naïve schizophrenia patient at the start of Olanzapine treatment and the results were promising in regards of weight loss as well as improvement in other metabolic parameters. The patients that were enrolled in the study were of normal body weight and body mass index during the study. These findings further strengthen the fact that the patient need not to be overweight to be a candidate for Topiramate therapy in patients receiving Olanzapine treatment.

Further discussing on Topiramate (TPM) treatment, TPM is an approved drug agent formally used in the prophylaxis of epilepsy and migraine is also known to block AMPA/kainate-gated ion and sodium channels and positively modulate GABA receptors. ${ }^{8}$ The proposed mechanism through which Topiramate 
exerts weight loss is through reduction in visceral fat associated with a decrease of plasma leptin level and increase in plasma adiponectin level, leading to an improvement in insulin sensitivity. ${ }^{9}$ Topiramate is reportedly found to reduce weight in the patients treated for seizure or for bipolar affective disorder. Its interest in the patients with binge eating disorder with obesity has also come up with effective results. ${ }^{10}$ In a study conducted by Van et al, ${ }^{11}$ Topiramate was used as a weight-loss agent for anxiety disorders patients receiving selective-serotonin reuptake inhibitors (SSRIs) and they reported that Topiramate could be a useful strategy to counteract SSRI related weight gain. Nickel et al, ${ }^{12}$ were the first to evaluate the role of Topiramate to attenuate atypical antipsychotic associated weight gain in 2005. And since then on, several studies have been conducted which suggests that Topiramate is an effective treatment for antipsychotic induced weight gain in patient with schizophrenia. ${ }^{3,10,13} \mathrm{Co}$-administration of Topiramate along with Olanzapine treatment have not only resulted in a reduction of Olanzapine induced weight gain and appetite, but have also resulted in improvement in insulin sensitivity and favorably decreased fasting blood glucose, total cholesterol, triglycerides, HDL cholesterol and leptin levels and that is also without aggravating the psychotic symptoms. ${ }^{3}$

Although the effect of Topiramate is found to be marvelous in reduction of antipsychotic induced weight gain, controlled studies, however, are limited by prescription of fixed doses (100-250 mg/day). In the 12 week, randomized, placebo controlled prospective study conducted in schizophrenia patient carrying excess weight, a higher dose of Topiramate at $200 \mathrm{mg}$ was found to be effective than $100 \mathrm{mg}$ in reducing antipsychotic induced weight gain.10 While in the retrospective chart review conducted to determine the efficacy and tolerability of longterm Topiramate treatment of psychotropic drug induced weight gain, the diminution of BMI was not correlated to Topiramate dosage; and moreover, this diminution was observed to be treatment duration dependent. 8 Hence, it can be noticed that there is a disagreement finding with that of a fixed-dose study
(100 vs. $200 \mathrm{mg} /$ day); specifically saying, superiority of a higher dosage. Although, the lack of association between BMI loss and Topiramate dosage observed in the retrospective chart review might be a consequence of the flexible and slow dosing schedule used, however, it does highlights a fact that there is a large variability in dose responses. The weight loss effect of Topiramate has been observed with a mean Topiramate dosage of $186.8 \pm 138.3 \mathrm{mg} /$ day, with a wide interindividual variability of doses (range $=25-800 \mathrm{mg}$ / day). ${ }^{8}$ Similarly, looking to the recent findings from the clinical trial conducted in healthy male volunteers in the Netherland, which demonstrates that the low basal TSH levels are associated with a relatively high body weight gain as a consequence of Olanzapine treatment, and the effectiveness of Topiramate in attenuating Olanzapine induced weight gain is also relatively higher in those subjects with a low basal TSH level; it can be noticed that there is interindividual differences in response to weight gain effect by Olanzapine treatment and weight loss effect by Topiramate treatment. If such is the case, it can be assumed that there might be a sub-set of individuals who might response to Topiramate treatment as low as the dose at $50 \mathrm{mg}$, more specifically saying; those individuals with low baseline TSH might have a good response at lower dose of Topiramate as low as at $50 \mathrm{mg}$. Hence, further rigorous studies to elaborate its effectiveness in weight reduction, mood stabilization and for control of seizure deems necessary.

\section{CONCLUSION}

Consistent supporting findings suggesting Topiramate's effectiveness and safety for weight reduction and for symptomatic improvement in patients with schizophrenia spectrum disorders; it can be argued that Topiramate can be used safely and effectively in patients with schizophrenia. It is concluded that Topiramate adjunctive therapy for Olanzapine induced weight gain would not only benefit at clinical level but will also improve at metabolic level thereby decreasing non compliance in this patient group. 


\section{REFERENCES}

1. Leucht S, Corves C, Arbter D, Engel RR, Li C, Davis JM. Second-generation versus first-generation antipsychotic drugs for schizophrenia: a meta-analysis. The Lancet .373(9657):31-41.

2. Keinänen J, Mantere $\mathrm{O}$, Kieseppä T, Mäntylä $\mathrm{T}$, Torniainen $\mathrm{M}$, Lindgren $\mathrm{M}$, et al. Early insulin resistance predicts weight gain and waist circumference increase in first-episode psychosis - A one year follow-up study. Schizophrenia Research. 2015;169(1-3):45863.

3. Narula PK, Rehan HS, Unni KES, Gupta N. Topiramate for prevention of olanzapine associated weight gain and metabolic dysfunction in schizophrenia: A doubleblind, placebo-controlled trial. Schizophrenia Research. 2010;118(1-3):218-23.

4. Kinon BJ, Kaiser CJ, Ahmed S, Rotelli MD, Kollack-Walker S. Association between early and rapid weight gain and change in weight over one year of olanzapine therapy in patients with schizophrenia and related disorders. Journal of clinical psychopharmacology. 2005;25(3):2558.

5. Maayan L, Vakhrusheva J, Correll CU. Effectiveness of medications used to attenuate antipsychotic-related weight gain and metabolic abnormalities: a systematic review and metaanalysis. Neuropsychopharmacology:official publication of the American College of Neuropsychopharmacology. 2010;35(7):152030.

6. Chang CT, Chen YC, Fang JT, Huang CC. Metformin-associated lactic acidosis: case reports and literature review. Journal of nephrology. 2002;15(4):398-402.

7. Chen Y, Zhou K, Wang R, Liu Y, Kwak YD, $\mathrm{Ma} \mathrm{T}$, et al. Antidiabetic drug metformin
(GlucophageR) increases biogenesis of Alzheimer's amyloid peptides via up-regulating BACE1 transcription. Proceedings of the National Academy of Sciences of the United States of America. 2009;106(10):3907-12.

8. Khazaal Y, Chatton A, Rusca M, Preisig M, Zullino D. Long-term topiramate treatment of psychotropic drug-induced weight gain: a retrospective chart review. General hospital psychiatry. 2007;29(5):446-9.

9. Mahmood S, Booker I, Huang J, Coleman CI. Effect of topiramate on weight gain in patients receiving atypical antipsychotic agents. Journal of clinical psychopharmacology. 2013;33(1):90-4.

10. Ko YH, Joe SH, Jung IK, Kim SH. Topiramate as an adjuvant treatment with atypical antipsychotics in schizophrenic patients experiencing weight gain. Clinical neuropharmacology. 2005;28(4):169-75.

11. Van Ameringen M, Mancini C, Pipe B, Campbell M, Oakman J. Topiramate treatment for SSRI-induced weight gain in anxiety disorders. The Journal of clinical psychiatry. 2002;63(11):981-4.

12. Nickel MK, Nickel C, Muehlbacher M, Leiberich PK, Kaplan P, Lahmann C, et al. Influence of topiramate on olanzapine-related adiposity in women: a random, double-blind, placebo-controlled study. Journal of clinical psychopharmacology. 2005;25(3):211-7.

13. Evers SS, van Vliet A, van Vugt B, Scheurink AJW, van Dijk G. A low TSH profile predicts olanzapine-induced weight gain and relief by adjunctive topiramate in healthy male volunteers. Psychoneuroendocrinology. 2016;66:101-10. 